Pacific Journal of Mathematic 


\section{ON THE STRUCTURE OF TOR, II}

\section{R. J. NUNKE}

The following results are proved:

If $A$ and $B$ are abelian $p$-groups and the length of $A$ is greater than the length of $B$, then Tor $(A, B)$ is a direct sum of countable groups if and only if (i) $B$ is a direct sum of countable groups and (ii) if the $\beta$-th Ulm invariant of $B$ is not zero, then every $p^{\beta} A$-high subgroup of $A$ is a direct sum of countable groups.

If $\beta$ is an ordinal, $A$ is a $p$-group, and if one $p^{\beta} A$-high subgroup of $A$ is a direct sum of countable groups then every $p^{\beta} A$-high subgroup of $A$ is a direct sum of countable groups.

If $A$ and $B$ are $p$-groups of cardinality $\leqq \aleph_{1}$ without elements of infinite height, then $\operatorname{Tor}(A, B)$ is a direct sum of cyclic groups.

For each $n$ with $1 \leqq n<\omega$, there is a $p$-group $G$ without elements of infinite height such that $G$ is not itself a direct sum of cyclic groups but every subgroup of $G$ having cardinality $\leqq \boldsymbol{H}_{n}$ is a direct sum of cyclic groups.

If $A$ and $B$ are (abelian) $p$-groups, when is Tor $(A, B)$ a direct sum of countable groups (d.s.c. group)? This paper contains a complete answer for this question when $A$ and $B$ have different lengths.

If $A$ and $B$ have the same length the situation is much more complicated. The simplest case occurs when $A$ and $B$ have no elements of infinite height. Then Tor $(A, B)$ has no elements of infinite height and it is a d.s.c. group if and only if it is a direct sum of cyclic groups ( $\Sigma$-cyclic). Here, although there is no satisfactory answer to the question, some partial results are obtained. For example it is shown that if $A$ and $B$ are $p$-groups without elements of infinite height having cardinalities $\leqq \mathcal{N}_{1}$, then Tor $(A, B)$ is $\Sigma$-cyclic.

Finally some examples of strange groups are constructed. Let a $p$-group be called $\lambda$-cyclic, where $\lambda$ is a cardinal number, if every subgroup with cardinality $<\lambda$ is $\Sigma$-cyclic. Every $p$-group without elements of infinite height is $\boldsymbol{\aleph}_{1}$-cyclic. For each $n$ with $1 \leqq n<\omega$, a group is constructed which is $\boldsymbol{\aleph}_{m}$-cyclic but not $\Sigma$-cyclic.

These results are obtained by homological methods together with the concept of $N$-high subgroup due to John Irwin [2].

In diagrams $>\longrightarrow$ denotes a monomorphism and $\longrightarrow>$ an epimorphism. An extension $C>\longrightarrow E \longrightarrow>A$ is $p^{\alpha}$-pure where $p$ is a prime and $\alpha$ an ordinal number if it belongs to $p^{\alpha} \operatorname{Ext}(A, C)$. A monomorphism $f: C>\longrightarrow E$ is $p^{\alpha}$-pure if the extension $C>\longrightarrow E \longrightarrow>$ Coker $f$ is. Similarly $C \subseteq E$ is a $p^{\alpha}$-pure subgroup of $E$ if the extension 
$C>\longrightarrow E \longrightarrow>E / C$ is $p^{\alpha}$-pure. If $E$ is a $p$-group, then $p^{\omega}$-purity coincides with the ordinary concept of purity. More generally, if $C>\longrightarrow E \longrightarrow>A$ is $p^{\alpha}$-pure then:

$\left(1^{\circ}\right) \quad\left(p^{\beta} A\right)[p]=\left(C+\left(p^{\beta} E\right)[p]\right) / C$ for all $\beta<\alpha$, and

$\left(2^{\circ}\right) \quad C \cap p^{\beta} E=p^{\beta} C$ for all $\beta \leqq \alpha$.

An easy transfinite induction shows that $\left(1^{\circ}\right) \Rightarrow\left(2^{\circ}\right)$. If $\alpha \leqq \omega$, then $\left(2^{\circ}\right) \Rightarrow p^{\alpha}$-purity. If $A$ is a divisible $p$-group then $\left(1^{\circ}\right) \Rightarrow p^{\alpha}$-purity for all ordinals $\alpha$. This last implication holds in certain other situations but not in general. These facts are proved in [8].

If $N$ is a subgroup of the group $G$, a subgroup $H$ of $G$ is called $N$-high in $G$ if $H$ is maximal with respect to the property $H \cap N=0$.

Proposition 1. If $G$ is a $p$-group, $N \cong p^{\alpha} G$, and $H$ is $N$-high in $G$, then $H$ is $p^{\alpha+1}$-pure in $G$. If $N \cong p^{\omega} G$, then $G / H$ is divisible. ${ }^{1}$

Proof. We prove first that if $G$ is a $p$-group, then $H$ is $N$-high in $G$ if and only if $H \cap N=0$ and $(G / H)[p]=(H+N[p]) / H$. To see this suppose $H$ is $N$-high in $G$. Clearly $H \cap N=0$ and $(H+N[p]) / H \subseteq(G / H)[p]$. Let $0 \neq x \in(G / H)[p]$ and let $g \in G$ map onto $x \bmod H$. Then $g \notin H, p g \in H$, and by the maximality of $H$ there is a nonzero $a \in H \cap(H+\{g\})$. Thus $a=h+k g$ with $k$ an integer. Moreover $p$ does not divide $k$ for otherwise $a \in H \cap N=0$. Hence $1=r k+s p$ for suitable integers $r$ and $s$ and $g=r k g+s p g=r a+$ $(s p g-r h)$. Now $s p g-r h \in H$ and $p r a \in G H \cap N=0$ so that $x \in(H+$ $N[p]) / H$ as desired.

Conversely suppose $(G / H)[p]=(H+N[p]) / H$ and $H \cap N=0$. To show the maximality of $H$ it is enough to show that $(H+\{g\} \cap N \neq 0$ whenever $g \notin H$ but $p g \in H$. If $g$ has these properties then the hypothesis gives $g=a+h$ with $a \in N$ and $h \in H$. Then $a \neq 0$ because $g \notin H$ and $a=g-h \in(H+\{g\}) \cap N$.

Now suppose $H$ is $N$-high in $G$ and $N \subseteq p^{\alpha} G$. Then by the result just proved we have $(G / H)[p] \subseteq\left(H+\left(p^{\beta} G\right)[p]\right) / H$ for all $\beta \leqq \alpha$. Since $\left(p^{\beta}(G / H)\right)[p] \subseteq(G / H)[p]$ and $\left.\left(H+\left(p^{\beta} G\right)[p]\right) / H \subseteq\left(p^{\beta} G / H\right)\right)[p]$ we have

$$
\left(p^{\beta}(G / H)\right)[p]=\left(H+\left(p^{\beta} G\right)[p]\right) / H \quad \text { for all } \beta \leqq \alpha .
$$

If $\alpha<\omega$ the discussion preceeding the statement of this proposition shows that $H$ is $p^{\alpha+1}$-pure in $G$. The same discussion shows $p^{\alpha+1}$-purity for $\alpha \geqq \omega$ once we know that $G / H$ is divisible.

If $N \subseteq p^{\omega} G$, then $(G / H)[p] \subseteq\left(H+p^{\omega} G\right) / H \subseteq p^{\omega}(G / H)$ which implis that $G / H$ is divisible.

Proposition 2. If $C>\longrightarrow E \longrightarrow A$ is $p^{\alpha}$-pure with $\alpha \geqq \omega$ and $B$ is any $p$-group, then

1 The first statement of this proposition and the first statement of [3] Theorem 2 read the same, however the term $p^{\alpha}$-purity has different meanings in the two places. 


$$
\text { Tor }(C, B)>\longrightarrow \text { Tor }(E, B) \longrightarrow>\text { Tor }(A, B)
$$

is exact and $p^{\alpha}$-pure.

Proof. The condition $\alpha \geqq \omega$ is needed only to show that Tor $(E, B) \rightarrow$ Tor $(A, B)$ is epic. We use the description of Tor $(A, B)$ in terms of generators and relations given in $[6, p .150]$. The generators are triples $\langle a, n, b\rangle$ with $n \in Z$ (the group if integers), $a \in A[n]$ and $b \in B[n]$. The relations require $\langle a, n, b\rangle$ to be bilinear as a function of $a$ and $b$ and also require $\langle k a, n, b\rangle=\langle a, k n, b\rangle$ for $k, n \in z, a \in A[k n], b \in B[n], \quad$ and $\langle a, n, k b\rangle=\langle a, k n, b\rangle$ for $k, n \in z$, $a \in A[n], b \in B[k n]$.

If $\alpha \geqq 0$, then $C>\longrightarrow E \longrightarrow A$ is $p^{\omega}$-pure and it follows that each $a \in A\left[p^{n}\right]$ can be lifted to an element $e \in E$ with the same order. Since $B$ is a $p$-group, Tor $(A, B)$ is generated by the elements $\left\langle a, p^{n}, b\right\rangle$ with $p^{n} a=0=p^{n} b$. Letting $e \in E\left[p^{n}\right]$ map onto $a$ we have $\left\langle e, p^{n}, b\right\rangle \in \operatorname{Tor}(E, B)$ mapping onto $\left\langle a, p^{n}, b\right\rangle$ as required to show that $\operatorname{Tor}(E, B) \rightarrow \operatorname{Tor}(A, B)$ is epic.

The sequence with Tor is now exact because Tor is left-exact.

For a given $\alpha$, the functor $p^{\alpha}$ is represented by an exact sequence $Z>\longrightarrow G \longrightarrow H$ (See [7] or [8] for the definitions and details). For a group $A$ let $\partial_{A}$ : Tor $(H, A) \rightarrow A$ be the connecting homomorphism induced by this sequence. We then have

$$
\partial_{A}\langle x, n, a\rangle=(n y) a
$$

where $y$ is any element of $G$ mapping onto $x$. Since $n x=0, n y \in Z$ so that the term $(n y) a$ makes sense.

The extension

$$
C>\longrightarrow E \stackrel{\lambda}{\longrightarrow}>A
$$

is $p^{\alpha}$-pure if and only if there is a map $\varphi$ : Tor $(H, A) \rightarrow E$ such that $\lambda \varphi=\partial_{A}$.

MacLane shows in [5] that, for groups $A, B, C$, the group Tor (Tor $(A, B), C)$ is generated by the elements $\langle\langle a, n, b\rangle, n, c\rangle$ with $n \in Z n a=n b=n c=0$. Similarly Tor $(A$, Tor $(B, C))$ is generated by the elements $\langle a, n,\langle b, n, c\rangle\rangle$. Moreover there is a natual isomorphism

$$
\theta: \text { Tor }(\operatorname{Tor}(A, B), C)=\operatorname{Tor}(A, \operatorname{Tor}(B, C))
$$

such that

$$
\theta\langle\langle a, n, b\rangle, n, c\rangle=\langle a, n,\langle b, n, c\rangle\rangle .
$$

For groups $A, B$ we have a diagram 


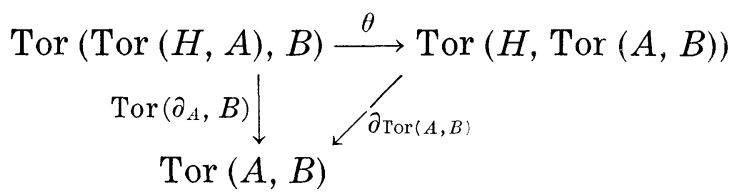

This diagram commutes for we have

$$
\begin{aligned}
\operatorname{Tor}\left(\partial_{A}, B\right)\langle\langle x, n, a\rangle, n, b\rangle & =\left\langle\partial_{A}\langle x, n, a\rangle, n, b\right\rangle \\
& =\langle(n y) a, n, b\rangle \\
& =(n y)\langle a, n, b\rangle \\
& =\partial_{\operatorname{Tor}(A, B)}\langle x, n,\langle a, n, b\rangle\rangle \\
& =\partial_{\operatorname{Tor}(A, B)} \theta\langle\langle x, n, a\rangle, n, b\rangle .
\end{aligned}
$$

Now suppose $C>\longrightarrow E \longrightarrow>A$ is $p^{\alpha}$-pure with $\lambda: E \rightarrow A$. Hence $\partial_{A}=\lambda \varphi$. Applying Tor we get Tor $\left(\partial_{A}, B\right)=\operatorname{Tor}(\lambda, B)$ Tor $(\varphi, B)$ and therefore $\partial_{\operatorname{Tor}(A, B)}=\operatorname{Tor}(\lambda, B)$ Tor $(\varphi, B) \theta^{-1}$. Thus the sequence

$$
\text { Tor }(C, B)>\longrightarrow \text { Tor }(E, B) \longrightarrow>\text { Tor }(A, B)
$$

is $p^{\alpha}$-pure.

For the purposes of this paper we define the length $\lambda(A)$ of the $p$-group $A$ to be the least ordinal $\alpha$ such that $p^{\alpha} A=0$ and $\infty$ if there is no such ordinal. The symbol $\infty$ is assumed to be larger than any ordinal. According to [7] $p^{\alpha}$ Tor $(A, B)=$ Tor $\left(p^{\alpha} A, p^{\alpha} B\right)$ so that the length of Tor $(A, B)$ is the minimum of the lengths of $A$ and of $B$. The group $A$ is $p^{\alpha}$-projective if each $p^{\alpha}$-pure extension $C>\longrightarrow E \longrightarrow A$ splits. A d.s.c. group is $p^{\alpha}$-projective if and only if it has length $\leqq \alpha$ ([7] or [8]).

In the proofs of the next few theorems we shall refer repeatedly to the following situation. Let $\beta$ be an infinite ordinal and let $M$ be a $p^{\beta} A$-high subgroup of $A$. Then by Proposition 1 the sequence

$$
M>\longrightarrow A \longrightarrow>A / M
$$

is $p^{\beta+1}$-pure and $A / M$ is divisible. If $B$ is a $p$-group, then by Proposition 2 the sequence

$(*) \quad \operatorname{Tor}(M, B)>\longrightarrow$ Tor $(A, B) \longrightarrow>\operatorname{Tor}(A / M, B)$

is also $p^{\beta+1}$-pure. Moreover if $A / M \neq 0$, then $A / M=\Sigma Z\left(p^{\infty}\right)$ and hence Tor $(A / M, B)=\Sigma$ Tor $\left(Z\left(p^{\infty}\right), B\right)=\Sigma B$.

In the remainder of the paper we shall use without further reference Kaplansky's theorem [4] that a direct summand of a d.s.c. group is itself one.

Proposition 3. If $\beta$ is an infinite countable ordinal, $A$ has a $p^{\beta} A$-high subgroup which is a d.s.c. group, and $B$ is a countable $p$-group of length $\leqq \beta+1$, then $\operatorname{Tor}(A, B)$ is a d.s.c. group. 
Proof. Let $M$ be the $p^{\beta} A$-high subgroup called for and refer to the $p^{\beta+1}$-pure sequence $\left(^{*}\right)$ above. Since $B$ is countable of length $\leqq \beta+1$ it is $p^{\beta+1}$-projective. Hence $\operatorname{Tor}(A / M, B)=\Sigma B$ is also $p^{\beta+1}$ projective and the sequence $\left(^{*}\right)$ splits. But then Tor $(A, B)$ is a direct sum of the d.s.c. groups Tor $(M, B)$ and $\operatorname{Tor}(A / M, B)$ and is therefore a d.s.c. group. $\operatorname{Tor}(M, B)$ is a d.s.c. group because $M$ is a d.s.c. group, Tor commutes with direct sums and $\operatorname{Tor}(G, B)$ is countable whenever $G$ and $B$ are.

Proposition 4. Let Tor $(A, B)$ be a d.s.c. group.

(i) If $\lambda(A)>\lambda(B)$, then $B$ is a d.s.c. group.

(ii) If $\lambda(A) \geqq \lambda(B)=\beta+1$ with $\beta$ an infinite countable ordinal, and $B$ is a d.s.c. group, then every $p^{\beta} A$-high subgroup of $A$ is a d.s.c. group.

Proof. To show (i) let $\beta=\lambda(B)$. If $\beta<\omega$ then $B$ has bounded order and is clearly a d.s.c. group. Hence suppose $\beta \geqq \omega$. Let $M$ be a $p^{\beta} A$-high subgroup of $A$ and consider the $p^{\beta+1}$-pure sequence $\left(^{*}\right)$. Since $\operatorname{Tor}(A, B)$ is a d.s.c. group of length $\beta$ it is $p^{\beta}$-projective. According to $\left[8\right.$, Proposition 3.1] the $p^{\beta+1}$-purity of $\left({ }^{*}\right)$ then implies that the sequence $\left(^{*}\right)$ splits. Hence $\operatorname{Tor}(A / M, B)$ is a d.s.c. group. Since $\lambda(A)>\beta, A / M \neq 0$ so that $B$ is a direct summand of Tor $(A / M, B)$ and therefore a d.s.c. group.

To prove (ii) we again let $M$ be $p^{\beta} A$-high and refer to (*). Now $B$ is $p^{\beta+1}$-projective so that Tor $(A, B)$ is $p^{\beta+1}$-projective. Hence the sequence $\left(^{*}\right)$ splits. Therefore Tor $(M, B)$ is a d.s.c. group. Since $\lambda(M)=\beta<\lambda(B), M$ is a d.s.c. group by (i) and the commutativity of Tor.

Corollary 5. If $\beta$ is an infinite ordinal and the p-group $A$ has one $p^{\beta} A$-high subgroup which is a d.s.c. group, then every $p^{\beta} A$-high subgroup of $A$ is a d.s.c. group.

Proof. If $\lambda(A) \leqq \beta$, then $A$ is the only $p^{\beta} A$-high subgroup so the result is trivial. Therefore assume $\lambda(A)>\beta$. Next observe that if $\beta>\Omega$, then a $p^{\beta} A$-high subgroup cannot be a d.s.c. group because it has length $\beta$ and the length of a d.s.c. group is either $\leqq \Omega$ or is $\infty$.

If $\beta=\Omega$ and $M$ is $p^{\beta} A$-high, then $M>\longrightarrow A \longrightarrow A / M$ is in $p^{\Omega} \operatorname{Ext}(A / M, M)$. If $M$ is a d.s.c. group, then $p^{\Omega} \operatorname{Ext}\left(Z\left(p^{\infty}\right), M\right)=0$ by [8] Lemma 3.10. Since $A / M=\Sigma Z\left(p^{\infty}\right)$,

$$
\operatorname{Ext}(A / M, M)=\Pi \operatorname{Ext}\left(Z\left(p^{\infty}\right), M\right)
$$

so that $p^{\Omega} \operatorname{Ext}(A / M, M)=0$. Thus $M$ is a direct summand of $A$. 
Since $M$ is $p^{\Omega} A$-high in $A$ we have $(A / M)[p] \cong\left(p^{\Omega} A\right)[p]$ and it follows easily that $p^{\Omega} A$ is the maximal divisible subgroup of $A$. Hence $M \cong$ $A / p^{\Omega} A$ in this case. Since $A / p^{g} A$ is independent of $M$ it follows that all $p^{\Omega} A$-high subgroups are isomorphic (if one is a d.s.c. group) and hence all are d.s.c. groups.

Finally if $\beta$ is infinite and countable, let $B$ be a countable $p$-group of length $\beta+1$. By Proposition 3 Tor $(A, B)$ is a d.s.c. group because $A$ has a $p^{\beta} A$-high subgroup which is a d.s.c. group. By Proposition 4 (ii) every $p^{\beta} A$-high subgroup of $A$ is a d.s.c. group.

Theorem 6. If $\lambda(A)>\lambda(B) \geqq \omega$, then Tor $(A, B)$ is a d.s.c. group if and only if

(i) $B$ is a d.s.c. group, and

(ii) if $\beta$ is an infinite ordinal such that the $\beta$-th Ulm invariant of $B$ is $\neq 0$, then every $p^{\beta} A$-high subgroup of $A$ is a d.s.c. group.

Proof. We need two easy consequences of Ulm's theorem and Zippin's theorem (cf. [1] p. 135):

(1) If $B$ is a d.s.c. group whose $\beta$-th Ulm invariant is not zero, then $B$ has a countable direct summand $B^{\prime}$ of length $\beta+1$.

(2) If $B$ is a d.s.c. group, then $B=\Sigma B_{i}$ where $i$ ranges over some index set and $B_{i}$ is countable of length $\beta_{i}+1$.

Suppose Tor $(A, B)$ is a d.s.c. group. We get (i) by proposition 4(i). Suppose further that $\beta$ is infinite and the $\beta$-th Ulm invariant of $B$ is not zero. Let $B^{\prime}$ be a countable direct summand of $B$ with length $\beta+1$ as provided by (1) above. Then Tor $\left(A, B^{\prime}\right)$ is a direct summand of Tor $(A, B)$, hence a d.s.c. group and (ii) follows from Proposition 4(ii).

Suppose $\lambda(A)>\lambda(B) \geqq \omega$ and (i) (ii) are satisfied. Using (2) above we write $B=\Sigma B_{i}$ with $B_{i}$ countable of length $\beta_{i}+1$. Then Tor $(A, B)=\Sigma$ Tor $\left(A, B_{i}\right)$. If $\beta_{i}<\omega$, then $B_{i}$ is a direct sum of cyclic groups so Tor $\left(A, B_{i}\right)$ is a d.s.c. group. If $\beta_{\imath} \geqq \omega$, then Tor $\left(A, B_{i}\right)$ is a d.s.c. group by Proposition 3. Hence Tor $(A, B)$ is a d.s.c. group.

In order to continue we must derive further properties of Tor. The inclusions $A^{\prime} \subseteq A, B^{\prime} \subseteq B$ induce a monomorphism Tor $\left(A^{\prime}, B^{\prime}\right)>\longrightarrow$ Tor $(A, B)$. We shall identify Tor $\left(A^{\prime}, B^{\prime}\right)$ with its image in Tor $(A, B)$.

\section{LEMMA 7.}

( i ) If $A^{\prime}, A^{\prime \prime} \leqq A$, then $\operatorname{Tor}\left(A^{\prime} \cap A^{\prime \prime}, B\right)=\operatorname{Tor}\left(A^{\prime}, B\right) \cap \operatorname{Tor}\left(A^{\prime \prime}, B\right)$.

(ii) If $A^{\prime} \subseteq A$ and $B^{\prime} \subseteq B$, then

$$
\operatorname{Tor}\left(A^{\prime}, B^{\prime}\right)=\operatorname{Tor}\left(A^{\prime}, B\right) \cap \operatorname{Tor}\left(A, B^{\prime}\right) .
$$


(iii) If $A^{\prime}, A^{\prime \prime} \cong A$ and $B^{\prime}, B^{\prime \prime} \supseteqq B$, then Tor $\left(A^{\prime} \cap A^{\prime \prime}, B^{\prime} \cap B^{\prime \prime}\right)=\operatorname{Tor}\left(A^{\prime}, B^{\prime}\right) \cap$ Tor $\left(A^{\prime \prime}, B^{\prime \prime}\right)$.

Proof. If $A^{\prime}, A^{\prime \prime} \leqq A$, then there is a commutative diagram

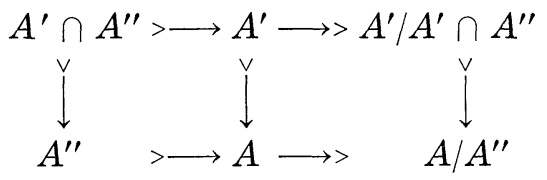

with exact rows and monic vertical maps. Applying Tor we get

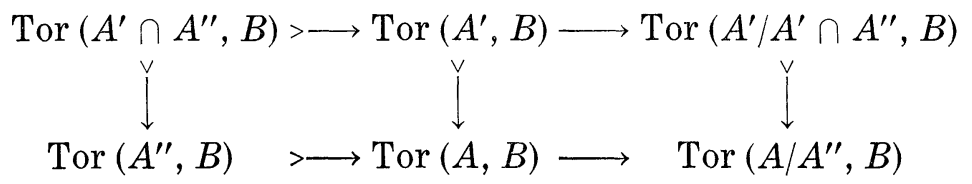

with exact rows and monic vertical maps. Conclusion (i) follows from this diagram.

To prove (ii) we note the existence of the commutative diagram

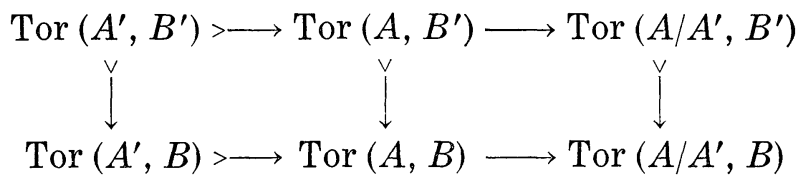

with exact rows and monic vertical maps and proceed as before.

For (iii) we have

$$
\begin{aligned}
\text { Tor } & \left(A^{\prime} \cap A^{\prime \prime}, B^{\prime} \cap B^{\prime \prime}\right) \\
& =\operatorname{Tor}\left(A^{\prime} \cap A^{\prime \prime}, B\right) \cap \operatorname{Tor}\left(A, B^{\prime} \cap B^{\prime \prime}\right) \\
& =\operatorname{Tor}\left(A^{\prime}, B\right) \cap \operatorname{Tor}\left(A^{\prime \prime}, B\right) \cap \operatorname{Tor}\left(A, B^{\prime}\right) \cap \operatorname{Tor}\left(A, B^{\prime \prime}\right) \\
& =\operatorname{Tor}\left(A^{\prime}, B^{\prime}\right) \cap \operatorname{Tor}\left(A^{\prime \prime}, B^{\prime \prime}\right)
\end{aligned}
$$

using in order (ii), (i), (ii).

Lemma 7 holds for any left exact covariant functor of two variables.

Proposition 8. For $x \in \operatorname{Tor}(A, B)$, there are unique finite subgroups $A_{x} \subseteq A$ and $B_{x} \subseteq B$ such that

(i) $x \in \operatorname{Tor}\left(A_{x}, B_{x}\right)$ and

(ii) if $x \in \operatorname{Tor}\left(A^{\prime}, B^{\prime}\right)$ with $A^{\prime} \subseteq A$ and $B^{\prime} \cong B$, then $A_{x} \leqq A^{\prime}$ and $B_{x} \subseteq B^{\prime}$.

Proof. There exist finite subgroups $G \subseteq A, H \cong B$ such that $x \in \operatorname{Tor}(G, H)$. Let $G_{1}, H_{1} ; G_{2}, H_{2} ; \cdots ; G_{n}, H_{n}$ enumerate the pairs of 
subgroups of $G$ and $H$ respectively such that $x \in \operatorname{Tor}\left(G_{i}, H_{i}\right)$ for $i=$ $1, \cdots, n$. By Lemma 7 (iii) we have $x \in$ Tor $\left(G_{1} \cap \cdots \cap G_{n}, H_{1} \cap \cdots \cap H_{n}\right)$. Put $A_{x}=G_{1} \cap \cdots \cap G_{n}$ and $B_{x} \in H_{1} \cap \cdots \cap H_{n}$. Thus (i) is satisfied. If $x \in \operatorname{Tor}\left(A^{\prime}, B^{\prime}\right)$ we have by Lemma 7 (iii) that

$$
x \in \operatorname{Tor}\left(G \cap A^{\prime}, H \cap B^{\prime}\right) .
$$

Then $G \cap A^{\prime}=G_{i}$ and $H \cap B^{\prime}=H_{i}$ for some $i$ so that $A_{x} \subseteq A^{\prime}$ and $B_{x} \subseteq B^{\prime}$ proving (ii).

Corollary 9. If $a \in A, b \in B$ have the same order and

$$
\text { Tor }(\{a\},\{b\}) \subseteq \text { Tor }\left(A^{\prime}, B^{\prime}\right)
$$

with $A^{\prime} \subseteq A$ and $B^{\prime} \leqq B$, then $a \in A^{\prime}$ and $b \in B^{\prime}$.

Proof. If the common order of $a$ and $b$ is $n$, then Tor $(\{a\},\{b\})$ is cyclic of order $n$. Let $x$ be a generator. Then $x \in \operatorname{Tor}\left(\{a\} \cup A^{\prime},\{b\} \cap B^{\prime}\right)$ by hypothesis and Lemma 7 (iii). If either $\{a\} \cap A^{\prime}$ or $\{b\} \cap B^{\prime}$ had order $<n$, then Tor $\left(\{a\} \cap A^{\prime},\{b\} \cap B^{\prime}\right)$ being cyclic would have order $<n$. Thus $x$ would have order $<n$ contradicting the fact that its order is $n$. It follows that $\{a\} \cap A^{\prime}=\{a\}$ and $\{b\} \cap B^{\prime}=\{b\}$ so that $a \in A^{\prime}$ and $b \in B^{\prime}$.

Proposition 10. If $A^{\prime} \subseteq A$ and $B^{\prime} \leqq B$ with $A, B p$-groups, $B^{\prime}$ has unbounded order, and Tor $\left(A^{\prime}, B^{\prime}\right)$ is pure in Tor $(A, B)$, then $A^{\prime}$ is pure in $A$.

Proof. Let $a \in A^{\prime} \cap p^{n} A$. Since $B^{\prime}$ has unbounded order there is a $b \in p^{n} B^{\prime}$ having the same order as $a$. In [7] it was shown that $p^{n} \operatorname{Tor}(A, B)=$ Tor $\left(p^{n} A, p^{n} B\right)$. Hence

$$
\begin{aligned}
\operatorname{Tor}(\{a\},\{b\}) & \cong \operatorname{Tor}\left(A^{\prime} \cap p^{n} A, B^{\prime} \cap p^{n} B\right) \\
& \cong \operatorname{Tor}\left(A^{\prime}, B^{\prime}\right) \cap p^{n} \text { Tor }(A, B) \\
& \supseteqq p^{n} \operatorname{Tor}\left(A^{\prime}, B^{\prime}\right)=\operatorname{Tor}\left(p^{n} A^{\prime}, p^{n} B^{\prime}\right) .
\end{aligned}
$$

By Corollary $9 a \in p^{n} A^{\prime}$. Since $a$ and $n$ were arbitrary we have $A^{\prime} \cap p^{n} A=p^{n} A^{\prime}$ for all $n$ and $A^{\prime}$ is pure in $A$.

An indexed family $\left\{A_{\alpha\}_{\alpha}>\rho}\right.$ of subgroups of $A$ will be called a sequence of subgroups if $\alpha$ ranges over the set of ordinals less then some ordinal $\rho$ and $A_{\alpha} \leqq A_{\beta}$ whenever $\alpha \leqq \beta<\rho$. If $\left\{A_{\alpha}\right\}_{\alpha<\rho}$ is a sequence of subgroups of $A$, then $\bigcup A_{\alpha}$ (the set theoretical union) is the subgroup generated by the $A_{\alpha}$.

Proposition 11. If $\left\{A_{\alpha}\right\}_{\alpha<\rho}$ and $\left\{B_{\alpha}\right\}_{\alpha<\rho}$ are sequences of subgroups of $A$ and $B$ respectively, then $\left\{\operatorname{Tor}\left(A_{\alpha}, B_{\alpha}\right)\right\}_{\alpha<\rho}$ is a sequence of 
subgroups of Tor $(A, B)$ and

$$
\bigcup \operatorname{Tor}\left(A_{\alpha}, B_{\alpha}\right)=\operatorname{Tor}\left(\bigcup A_{\alpha}, \bigcup B_{\alpha}\right) \text {. }
$$

Proof. It is clear that $\left\{\operatorname{Tor}\left(A_{\alpha}, B_{\alpha}\right)\right\}_{\alpha<\rho}$ is a sequence of subgroups of Tor $(A, B)$. Since $A_{\alpha} \leqq \bigcup A_{\alpha}$ and $B_{\alpha} \leqq \bigcup B_{\alpha}$ for all $\alpha<\rho$ we have

$$
\text { U Tor }\left(A_{\alpha}, B_{\alpha}\right) \leqq \operatorname{Tor}\left(\bigcup A_{\alpha}, \bigcup B_{\alpha}\right) \text {. }
$$

Suppose $x \in$ Tor $\left(\bigcup A_{\alpha}, \bigcup B_{\alpha}\right)$ and let $A_{x}, B_{x}$ be the subgroups defined

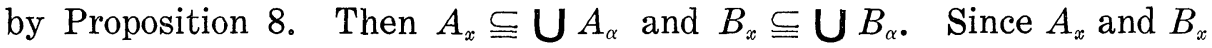
are finite, there is a $\beta<\rho$ such that $A_{x} \subseteq A_{\beta}$ and $B_{x} \subseteq B_{\beta}$. Hence $x \in \operatorname{Tor}\left(A_{\beta}, B_{\beta}\right) \leqq \mathrm{U} \operatorname{Tor}\left(A_{\alpha}, B_{\alpha}\right)$.

By the term $\Sigma$-cyclic we shall mean a direct sum of cyclic groups. A $p$-group is $\Sigma$-cyclic if and only if it is a d.s.c. group without elements of infinite height. In view of Proposition 4 if Tor $(A, B)$ is $\Sigma$-cyclic and $A$ has elements of infinite height, then $B$ is $\Sigma$-cyclic.

The following theorem gives a necessary condition for Tor $(A, B)$ to be $\Sigma$-cyclic. The symbol $|A|$ denotes the cardinality of $A$.

Theorem 12. If Tor $(A, B)$ is $\Sigma$-cyclic and $B$ is not $\Sigma$-cyclic, then

(i) $p^{\omega} A=0$, and

(ii) if $A^{\prime} \leqq A$ with $\left|A^{\prime}\right| \geqq|B|$, then $A^{\prime}$ is contained in a pure subgroup $A^{\prime \prime}$ of the same cardinality, such that $p^{\omega}\left(A / A^{\prime \prime}\right)=0$ and Tor $\left(A / A^{\prime \prime}, B\right)$ is $\Sigma$-cyclic.

Proof. As stated above conclusion (i) follows from Proposition 4.

Recall that if $A$ and $B$ are infinite p-groups, then $|\operatorname{Tor}(A, B)|=$ $|A||B|$. Now let $A^{\prime} \subseteq A$ with $\left|A^{\prime}\right| \geqq|B|$ and let Tor $(A, B)=\Sigma C_{\text {。 }}$ with the sum direct and each $C_{i}$ cyclic.

If $G$ is an infinite subgroup of Tor $(A, B)$, then, since each element has nonzero component in but a finite number of the summands $C_{i}$, $G$ is contained in a subgroup $G^{\prime}=\sum_{j \in J} C_{j}$ where $J$ is a subset of the index set and $\left|G^{\prime}\right|=|G|$. Moreover there are subgroups $A_{0} \sqsubseteq A$, $B_{0} \subseteq B$ such that $\left|A_{0}\right|=\left|B_{0}\right|=|G|$ and $G \subseteq$ Tor $\left(A_{0}, B_{0}\right)$. This is so because each $x \in \operatorname{Tor}(A, B)$ is a finite sum of elements of the form $\langle a, n, b\rangle$.

We define recursively a sequence

$$
\begin{aligned}
\operatorname{Tor}\left(A^{\prime}, B\right) & \subseteq G_{1} \leqq \operatorname{Tor}\left(A_{1}, B\right) \leqq G_{2} \leqq \cdots \\
& \cong G_{n} \leqq \operatorname{Tor}\left(A_{n}, B\right) \subseteq G_{n+1} \leqq \cdots
\end{aligned}
$$

of subgroups all having the same cardinality such that $G_{n}$ is the sum of a set of the $C_{i}$ appearing in the chosen direct sum decomposition of $\operatorname{Tor}(A, B)$. 
Let $A^{\prime \prime}=\bigcup A_{n}$. Then by Proposition 11,

$$
\text { Tor }\left(A^{\prime \prime}, B\right)=\operatorname{Tor} \bigcup\left(A_{n}, B\right)=\bigcup G_{n} .
$$

Hence Tor $\left(A^{\prime \prime}, B\right)$ is the sum of a set of the $C_{i}$ and is therefore a direct summand of Tor $(A, B)$. Hence Tor $\left(A^{\prime \prime}, B\right)$ is pure in Tor $(A, B)$. Since $B$ is not $\Sigma$-cyclic, it is unbounded and $A^{\prime \prime}$ is pure in $A$ by Proposition 10. Hence the sequence

$$
\text { Tor }\left(A^{\prime \prime}, B\right)>\longrightarrow \text { Tor }(A, B) \longrightarrow>\text { Tor }\left(A / A^{\prime \prime}, B\right)
$$

is exact and splits. Thus Tor $\left(A / A^{\prime \prime}, B\right)$ is $\Sigma$-cyclic and $p^{\omega}\left(A / A^{\prime \prime}\right)=0$ by part (i).

CoRollary 13. If $A$ and $B$ are p-groups without elements of infinite height, $B$ is not $\Sigma$-cyclic, $|A|>|B|$, and $A$ has greater cardinality than a basic subgroup, then Tor $(A, B)$ is not $\Sigma$-cyclic.

Proof. Suppose Tor $(A, B)$ is $\Sigma$-cyclic and let $C$ be a basic subgroup of $A$ such that $|C|<|A|$. There is a subgroup $A^{\prime}$ with $C \leqq A^{\prime} \subseteq A$ and $|A|>\left|A^{\prime}\right| \geqq|B|$. By Theorem 12 there is a subgroup $A^{\prime \prime}$ with $A^{\prime} \subseteq A^{\prime \prime} \subseteq A,\left|A^{\prime \prime}\right|=\left|A^{\prime}\right|$, and $p^{\omega}\left(A / A^{\prime \prime}\right)=0$. Now $A / A^{\prime \prime}$ is divisible because $C \cong A^{\prime \prime}$ and $A / C$ is divisible. Moreover $A / A^{\prime \prime} \neq 0$ because $\left|A^{\prime \prime}\right|<|A|$. Hence $p^{\omega}\left(A / A^{\prime \prime}\right) \neq 0$ contradicting the construction of $A^{\prime \prime}$. Therefore Tor $(A, B)$ is not $\Sigma$-cyclic.

LEMma 14. Let $A$ be a p-group and let $\rho$ be the least ordinal having the same cardinality as $|A|$. Then there is a sequence $\left\{A_{\alpha}\right\}_{\alpha<\rho}$ of subgroups of $A$ such that $\bigcup_{\alpha<\rho} A_{\alpha}=A$ and

(i) each $A_{\alpha}$ is pure in $A$,

(ii) $A_{\alpha}=\bigcup_{\beta<\alpha} A_{\beta}$ if $\beta$ is a limit ordinal $<\rho$,

(iii) $\left|A_{\alpha}\right|=\aleph_{0}$ if $\alpha<\omega$, and

(iv) $\left|A_{\alpha}\right|=|\alpha|$ if $\omega \leqq \alpha<\rho$.

Proof. Well order $A$ as $\left\{a_{\alpha}\right\}_{\alpha<\rho}$. Let $A_{0}$ be a countable pure subgroup of $A$ containing $a_{0}$. Suppose $A_{\beta}$ has been defined for all $\beta<\alpha$ satisfying (i)-(iv) above and also (v) $a_{r} \in A_{\beta}$ if $\gamma<\beta$ and $\beta$ is a singular ordinal $<\alpha$. If $\alpha$ is a limit ordinal (ii) forces the definition $A_{\alpha}=\bigcup_{\beta<\alpha} A_{\beta}$. Then (i)-(v) follow easily. If $\alpha=\gamma+1$, then $A_{\gamma}+$ $\left\{a_{r}\right\}$ has the same cardinality as $A_{\gamma}$ and there is a pure subgroup $A_{\alpha}$ of $A$ having the same cardinality as $A_{r}+\left\{a_{r}\right\}$. Then (i)-(v) are still satisfied. If $\alpha<\rho$, then $\left|A_{\alpha}\right|=|\alpha|<|A|$ so that the construction can be continued as long as $\alpha<\rho$. Since $a_{\alpha} \in A_{\alpha+1}$ it follows that $\mathrm{U}_{\alpha<\rho} A_{\alpha}=A$. 
THEOREM 15. If $A$ and $B$ are p-groups with the same cardinality such that every subgroup of either with smaller cardinality is a $\Sigma$-group, then Tor $(A, B)$ is $\Sigma$-cyclic.

Proof. Let $\left\{A_{\alpha}\right\}_{\alpha<\rho},\left\{B_{\alpha}\right\}_{\alpha<\rho}$, be sequences of subgroups of $A$ and of $B$ satisfying the conditions of Lemma 14. Since $\left|A_{\alpha}\right|=\left|B_{\alpha}\right|<|A|$ we have $A_{\alpha}$ and $B_{\alpha} \quad \Sigma$-cyclic for all $\alpha<\rho$. Set $G_{\alpha}=\operatorname{Tor}\left(A_{\alpha}, B_{\alpha}\right)$. Since $A_{\alpha}$ is pure in $A$ and $B_{\alpha}$ pure in $B, G_{\alpha}$ is pure in Tor $(A, B)$. Moreover by Proposition 11 and Lemma 14 (ii), $G_{\alpha}=\bigcup_{\beta<\alpha} G_{\beta}$ whenever $\alpha$ is a limit ordinal $<p$.

Since Tor is left exact, there is an exat sequence

$$
G_{\alpha}>\longrightarrow G_{\alpha+1} \longrightarrow \operatorname{Tor}\left(A_{\alpha+1} / A_{\alpha}, B_{\alpha+1}\right) \oplus \operatorname{Tor}\left(A_{\alpha+1}, B_{\alpha+1} / B_{\alpha}\right) \text {. }
$$

The term on the right is $\Sigma$-cyclic because $A_{\alpha+1}$ and $B_{\alpha+1}$ are $\Sigma$-cyclic. Thus $G_{\alpha+1} / G_{\alpha}$ is $\Sigma$-cyclic and therefore $G_{\alpha+1}=G_{\alpha} \oplus C_{\alpha}$ with $C_{\alpha} \Sigma$-cyclic because $G_{\alpha}$ is pure in $G_{\alpha+1}$. Hence we have a sequence $\left\{G_{\alpha}\right\}_{\alpha>\rho}$ of subgroups of Tor $(A, B)$ such that

$\left(1^{\circ}\right) \quad G_{\alpha} \subseteq G_{\alpha+1}$ for $\alpha<\rho$ and $G_{\alpha}=\bigcup_{\beta<\alpha} G_{\beta}$ if $\alpha$ is a limit ordinal $<\rho$;

$\left(2^{\circ}\right) \quad G_{\alpha+1}=G_{\alpha} \oplus C_{\alpha}$ with $C_{\alpha}$ a $\Sigma$-group for all $\alpha<\rho$;

(iii) $\operatorname{Tor}(A, B)=\bigcup_{\alpha<\rho} G_{\alpha}$.

It follows that $\operatorname{Tor}(A, B)=\Sigma C_{\alpha}$ and is therefore a $\Sigma$-group.

Corollary 16. If $A$ and $B$ are p-groups without elements of infinite height whose cardinality is at most $\boldsymbol{\aleph}_{1}$, then $\operatorname{Tor}(A, B)$ is s-cyclic.

If $\rho$ is a cardinal number, call a $p$-group $A \rho$-cyclic if every subgroup of cardinal $<\rho$ is $\Sigma$-cyclic. Every $p$-group without elements of infinite height is $\aleph_{1}$-cyclic. Let $\rho^{+}$be the cardinal next larger than $\rho$.

Corollary 17. If $A$ and $B$ are $\rho$-cyclic, then $\operatorname{Tor}(A, B)$ is a $\rho^{+}$-cyclic.

Proof. Let $G \leqq \operatorname{Tor}(A, B)$ with $|G|=\rho$. Since, for $x \in \operatorname{Tor}(A, B)$, there are finite subgroups $A^{\prime}, B^{\prime}$ of $A$ and $B$ respectively with $x \in \operatorname{Tor}\left(A^{\prime}, B^{\prime}\right)$, there are subgroups $A_{0} \subseteq A, B_{0} \subseteq B$ such that $\left|A_{0}\right|=$ $\left|B_{0}\right|=|G|=\rho$ and $G \cong$ Tor $\left(A_{0}, B_{0}\right)$. Since every subgroup of a direct sum of cyclic groups is one, $A_{0}$ and $B_{0}$ satisfy the hypotheses of Theorem 15. Hence Tor $\left(A_{0}, B_{0}\right)$ and therefore $G$ is $\Sigma$-cyclic. However $G$ was arbitrary with $|G|=\rho$ so the corollary follows.

For groups $A_{1}, \cdots, A_{n}$, define Tor $\left(A_{1}, \cdots, A_{n}\right)$ inductively as Tor $\left(\right.$ Tor $\left.\left(A_{1}, \cdots, A_{n-1}\right), A_{n}\right)$. 
LEMMA 18. If $A_{1}, \cdots, A_{2}$ are p-grrups without elements of infinite height, then Tor $\left(A_{1}, \cdots, A_{2}\right)$ is $\aleph_{n+1}$-cyclic.

Proof. The proof is by induction. We use the associativity of Tor to show that

$$
\operatorname{Tor}\left(A_{1}, \cdots, A_{2^{n}}\right)=\operatorname{Tor}\left(\operatorname{Tor}\left(A_{1}, \cdots, A_{m}\right), \operatorname{Tor}\left(A_{m+1}, \cdots, A_{2^{n}}\right)\right)
$$

where $m=2^{n-1}$ and then use Theorem 15 to complete the inductive step.

Proposition 19. For each $n$ with $1 \leqq n<\omega$, there is a $p$-group $G_{n}$ without elements of infinite height such that $G_{n}$ is $\boldsymbol{S}_{n}$-cyclic but not $\Sigma$-cyclic.

Proof. If $C$ is the direct sum of $\rho$ copies of $\Sigma Z\left(p^{n}\right)$ and $\rho \geqq \boldsymbol{\aleph}_{n}$, then the torsion completion of $C$ has cardinality $\rho^{\boldsymbol{\aleph}_{0}}$. Hence if $\rho^{\boldsymbol{N}_{0}}>\rho$, there is a $p$-group without elements of infinite height which has greater cardinality than a basic subgroup. Since there are arbitrarily large cardinals with this property there exists a sequence $A_{1}, A_{2}, \cdots, A_{n}, \cdots$ of $p$-groups of increasing cardinality, all without elements of infinite height, and all with greater cardinality than a basic subgroup.

Set $G_{n}=\operatorname{Tor}\left(A_{1}, \cdots, A_{2^{n-1}}\right)$ and $G_{1}=A_{1}$. Then $G_{n}$ is $\boldsymbol{N}_{n}$-cyclic by Lemma 18. If $A$ and $B$ are infinite torsion groups $\mid$ Tor $(A, B) \mid==$ $\max \{|A|,|B|\}$ so that $\left|\operatorname{Tor}\left(A_{1}, \cdots, A_{k}\right)\right|=\left|A_{k}\right|$. Thus $G_{n}$ is not $\Sigma$-cyclic by Theorem 15 .

\section{REFERENCES}

1. L. Fuchs, Abelian Groups, Budapest, 1958.

2. J. M. Irwin, High subgroups of abelian torsion groups, Pacific J. Math. 11 (1961), $1375-1384$.

3. J. M. Irwin and E. A. Walker, On isotype subgroups of abelian groups, Bull. Soc. Math. de France 89 (1961), 451-460.

4. I. Kaplansky, Projective modules, Ann. of Math. 68 (1958), 371-377.

5. S. MacLane, Triple torsion products and multiple Kunneth formulas, Math. Ann. 140 (1960), 51-64.

6. - Homology, Berlin, 1963.

7. R. J. Nunke, On the structure of Tor, Proceedings of the Colloquium on Abelian groups, Budapest (1964), 115-124.

8. - Homology and direct sums of countable abelian groups (to appear)

Received June 8, 1966. This work was supported in part by the National Science Foundation grant GP-5691. 


\section{PACIFIC JOURNAL OF MATHEMATICS}

\section{EDITORS}

\section{H. SAMELSON}

Stanford University

Stanford, California

J. P. JANS

University of Washington

Seattle, Washington 98105
J. DUGUNDJI

University of Southern California Los Angeles, California 90007

RICHARD ARENS

University of California

Los Angeles, California 90024

\section{ASSOCIATE EDITORS}
E. F. BECKENBACH
B. H. NEumanN
F. WOLF
K. YosidA

\section{SUPPORTING INSTITUTIONS}

\author{
UNIVERSITY OF BRITISH COLUMBIA \\ CALIFORNIA INSTITUTE OF TECHNOLOGY \\ UNIVERSITY OF CALIFORNIA \\ MONTANA STATE UNIVERSITY \\ UNIVERSITY OF NEVADA \\ NEW MEXICO STATE UNIVERSITY \\ OREGON STATE UNIVERSITY \\ UNIVERSITY OF OREGON \\ OSAKA UNIVERSITY \\ UNIVERSITY OF SOUTHERN CALIFORNIA
}

\author{
STANFORD UNIVERSITY \\ UNIVERSITY OF TOKYO \\ UNIVERSITY OF UTAH \\ WASHINGTON STATE UNIVERSITY \\ UNIVERSITY OF WASHINGTON \\ AMERICAN MATHEMATICAL SOCIETY \\ CHEVRON RESEARCH CORPORATION \\ TRW SYSTEMS \\ NAVAL ORDNANCE TEST STATION
}

Mathematical papers intended for publication in the Pacific Journal of Mathematics should be typewritten (double spaced). The first paragraph or two must be capable of being used separately as a synopsis of the entire paper. It should not contain references to the bibliography. Manuscripts may be sent to any one of the four editors. All other communications to the editors should be addressed to the managing editor, Richard Arens at the University of California, Los Angeles, California 90024.

50 reprints per author of each article are furnished free of charge; additional copies may be obtained at cost in multiples of 50 .

The Pacific Journal of Mathematics is published monthly. Effective with Volume 16 the price per volume ( 3 numbers) is $\$ 8.00$; single issues, $\$ 3.00$. Special price for current issues to individual faculty members of supporting institutions and to individual members of the American Mathematical Society: $\$ 4.00$ per volume; single issues $\$ 1.50$. Back numbers are available.

Subscriptions, orders for back numbers, and changes of address should be sent to Pacific Journal of Mathematics, 103 Highland Boulevard, Berkeley 8, California.

Printed at Kokusai Bunken Insatsusha (International Academic Printing Co., Ltd.), 7-17, Fujimi 2-chome, Chiyoda-ku, Tokyo, Japan.

PUBLISHED BY PACIFIC JOURNAL OF MATHEMATICS, A NON-PROFIT CORPORATION

The Supporting Institutions listed above contribute to the cost of publication of this Journal, but they are not owners or publishers and have no responsibility for its content or policies. 


\section{Pacific Journal of Mathematics

Vol. 22, No. $3 \quad$ March, 1967

Wai-Mee Ching and James Sai-Wing Wong, Multipliers and $H^{*}$

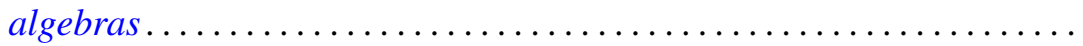

P. H. Doyle, III and John Gilbert Hocking, A generalization of the Wilder

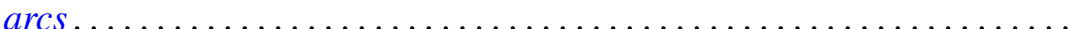

Irving Leonard Glicksberg, A Phragmén-Lindelöf theorem for function

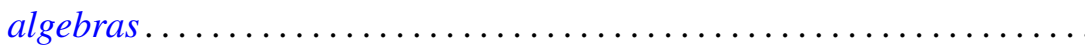

E. M. Horadam, A sum of a certain divisor function for arithmetical semi-groups ..................................... 407

V. Istrăţescu, On some hyponormal operators ................... 413

Harold H. Johnson, The non-invariance of hyperbolicity in partial

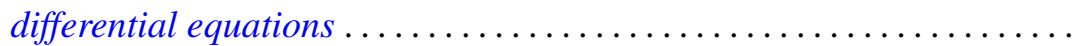

Daniel Paul Maki, On constructing distribution functions: A bounded denumerable spectrum with $n$ limit points................... 431

Ronald John Nunke, On the structure of Tor. II .................... 453

T. V. Panchapagesan, Unitary operators in Banach spaces ............. 465

Gerald H. Ryder, Boundary value problems for a class of nonlinear differential equations ................................. 477

Stephen Simons, The iterated limit condition and sequential convergence .................................... 505

Larry Eugene Snyder, Stolz angle convergence in metric spaces ......... 515 Sherman K. Stein, Factoring by subsets ................... 523

Ponnaluri Suryanarayana, The higher order differentiability of solutions of abstract evolution equations . . .

Leroy J. Warren and Henry Gilbert Bray, On the square-freeness of Fermat and Mersenne numbers ............................... 563

Tudor Zamfirescu, On l-simplicial convexity in vector spaces........... 565 Eduardo H. Zarantonello, The closure of the numerical range contains the spectrum 